GLOMEIRIC AND ALGEBRAIC TOPOLOGY

BANAC'H C'FNTER PUBLICATIONS, VOLUME I\&

PWN - POLISH SCIENTIFIC PUBLISHERS

WARSZAWA 1986

\title{
APPROXIMATIVE EXPANSIONS OF MAPS INTO INVERSE SYSTEMS*
}

\author{
TADASHI WATANABE \\ Yamagushi, Japan
}

\section{§1. Introduction}

We assume that the reader is familiar with the notions and terminlogy of inverse systems, maps between inverse systems (system maps), inverse limits etc., as in Eilenberg and Steenrod [13].

In [14] Freudenthal proved the following:

(1.1) Any compact metric space $X$ admits an inverse sequence $X$ $=\left\{X_{i}, p_{i j}, N\right\}$ such that $X=\lim X$ and all $X_{i}$ are finite polyhedra. Here the index set $N$ is the set of all positive integers.

(1.1) is important because it gives us a method to investigate $X$ by means of a polyhedral inverse sequence $X$. This fruitfull idea goes back to Alexandroff, Lefschetz and Freudenthal. Naturally, a question arises if one can apply this idea also to maps. If one attemps to do so, one encounters some difficulties. Most important are the following two questions: Let $X$ and $Y$ be compact metric spaces, let $X=\left\{X_{i}, p_{i j}, N\right\}$ and $Y=\left\{Y_{i}, g_{i j}, N\right\}$ be inverse sequences such that $X=\lim X, Y=\lim Y$, and let all $X_{i}$ and $Y_{i}$ be finite polyhedra.

(1.2) Given a map $f: X \rightarrow Y$, is there a system map $f: X \rightarrow Y$, for some $\boldsymbol{X}$ and $Y$, such that $f=\lim f$ ?

(1.3) Given a map $f: X \rightarrow Y$, is there a system map $f: X \rightarrow Y$ for any $X$ and $Y$ such that $f=\lim f$ ?

We will discuss the above questions on some examples. First, let $C$ and $I$ be the Cantor discontinuum and the unit interval, respectively. There is an onto map $F: C \rightarrow I$. Let $C=\left\{C_{i}, p_{i j}, N\right\}$ and $I=\left\{I_{i}, q_{i j}, N\right\}$ be inverse sequences such that $C=\lim C, I=\lim I$, all $C_{i}$ are finite sets, all $I_{i}$ are copies

* The detailed version of this paper will appear in Tsukuba J. Math. 
of $I$, and all $q_{i j}$ coincide with the identity map $1_{I}$. Let $\boldsymbol{p}=\left\{p_{i}: i \in N\right\}: C \rightarrow \boldsymbol{C}$ be an inverse limit and let all $q_{i}: I \rightarrow I_{i}$ coincide with $1_{1}$. Then $q$ $=\left\{q_{i}: i \in N\right\}: I \rightarrow I$ is also an inverse limit.

We first consider question (1.3). We assume that there is a system map $\boldsymbol{f}=\left\{f, f_{i}: i \in N\right\}: C \rightarrow \boldsymbol{I}$ such that $f=\lim \boldsymbol{f}$. Then for each $i \in N$ we have $q_{i} f=f_{i} p_{f(i)}$. Since $q_{i}$ and $f$ are onto, $f_{i}$ must also be onto. Then $f_{i}\left(C_{f(i)}\right)=I_{i}$ is a finite set, because $C_{f(i)}$ is finite. Hovewer, this is a contradiction. Hence, such a system map does not exist. This shows that in general the answer to (1.3) is negative.

Next, we will consider question (1.2). Let $K$ be an absolute retract (AR) which contains $C$ and $I$. In general we can take for $K$ the Hilbert cube $Q$. However, we can also take $I$ for $K$. Since $K$ is an AR, there exists a map $\bar{f}: K \rightarrow K$ such that $f=\bar{f} \mid C$. Take neighborhood systems $\left\{U_{i}: i \in N\right\}$ and $\left\{V_{i}: i \in N\right\}$ such that $U_{i+1} \subset U_{i}, V_{i+1} \subset V_{i}$ and $\bar{f}\left(U_{i}\right) \subset V_{i}$ for each $i \in N$, and $\bigcap\left\{U_{i}: i \in N_{j}=C\right.$ and $\cap\left\{V_{i}: i \in N\right\}=I$. Put $X_{i}=U_{i}, Y_{i}=V_{i}, f_{i}=\bar{f} \mid U_{i}: U_{i}$ $\rightarrow V_{i}$ for each $i \in N$, and let $p_{i j}$ and $q_{i j}$ be inclusions. Then $f$ $=\left\{1_{N}, f_{i}: i \in N\right\}: X=\left\{X_{i}, p_{i j}, N\right\} \rightarrow Y=\left\{Y_{i}, q_{i j}, N\right\}$ has the required properties. In general, by using inverse sequences of ANRs we obtain an affirmative answer for (1.2).

Many mathematicians have observed these phenomena. Freudenthal [14] and Mioduszewski [26] discussed question (1.3) and tried to expand maps into inverse sequences using some approximations. Especially, Mioduszewski [26] showed the existence of approximative expansions of maps into inverse sequences. In the next section we shall describe our method to deal with problems (1.2) and (1.3) which is natural and categorical. In order to do so we need some ideas and notions, which were developed in shape theory. Mardešić and Segal [22] is a good textbook for this theory.

\section{§. Approximative shape}

We will now introduce an approximative shape category and state without prool theorems. Detailed proofs can be found in Watanabe [31]

By a covering of a space $X$ we always mean a normal open covering. By $\operatorname{Cov}(X)$ we mean the set of all coverings of $X$. For $\mathscr{U}, \mathscr{U}^{\prime} \in \operatorname{Cov}(X)$, $\mathscr{U}$ $\geqslant$ ' $U$ ' means that ' $U^{\prime}$ is a refinement of ' $U$. Let $f, g: X \rightarrow Y$ be maps and let $\mathscr{V} \in \operatorname{Cov}(Y)$. We say that $f$ and $g$ are $\mathscr{V}$-near, in notation $(f, g) \leqslant \mathscr{V}$, provided for each $x \in X$ there is $V \in \mathscr{V}$ such that $f(x), g(x) \in V$. By $f^{-1}(\mathscr{V})$ we mean the covering $\left\{f^{-1}(V): V \in \mathscr{V}\right\}$ of $X$.

We say that $(X, \mathscr{U})=\left\{\left(X_{a}, \mathscr{U}_{a}\right), p_{a^{\prime}, a}, A\right\}$ is an approximative inverse system provided it satisfies the following three conditions:

(AI1) $X=\left\{X_{a}, p_{a^{\prime}, a}, A\right\}$ is an inverse system in Top where the index set $A$ is closure finite and directed and Top is the category of all spaces and all maps. 
(AI2) For each $a \in A$, $\mathscr{U}_{a}$ is a covering of $X_{\mathfrak{a}}$ such that for each $a^{\prime} \geqslant a$, $p_{a^{\prime}, a}^{-1}\left(\mathbb{M}_{a}\right) \geqslant \mathbb{M}_{a^{\prime}}$,

(AI3) For each $a \in A$ and for each $\mathscr{U} \in \operatorname{Cov}\left(X_{a}\right)$ there is an $a^{\prime} \geqslant a$ such that $p_{a^{\prime}, a}^{-1}(\mathscr{U}) \geqslant \mathscr{U}_{a^{\prime}}$.

A directed set $(A ; \geqslant)$ is called closure finite provided for each $a \in A$, $\left\{a^{\prime} \in A: a \geqslant a^{\prime}\right\}$ is a finite set.

Let $(\boldsymbol{Y}, \mathscr{V})=\left\{\left(Y_{b}, \mathscr{Y}_{b}\right), q_{b^{\prime}, b}, B\right\}$ be an approximative inverse system. We say that $\boldsymbol{f}=\left\{f, f_{b}: b \in \boldsymbol{B}\right\}:(\boldsymbol{X}, \mathscr{l}) \rightarrow(\boldsymbol{Y}, \mathscr{W})$ is an approximative system map provided $f: B \rightarrow A$ is a function and $f_{b}: X_{f(b)} \rightarrow Y_{b}$ are maps satisfying the following two conditions:

(AM1) $f_{b}^{-1}\left(\mathscr{V}_{b}\right) \geqslant \mathscr{U}_{f(b)}$ for each $b \in B$.

(AM2) For each $b^{\prime} \geqslant b$ there is an $a \geqslant f(b), f\left(b^{\prime}\right)$ such that $\left(f_{b} p_{a, f(b)}, q_{b^{\prime}, b} f_{b^{\prime}} \cdot p_{a \cdot f\left(b^{\prime}\right)}\right) \leqslant \mathscr{Y}_{h}$.

Let $\boldsymbol{f}=\left\{\bar{f}, \bar{f}_{b}: b \in \boldsymbol{B}\right\}:(\boldsymbol{X}, \boldsymbol{U}) \rightarrow(\boldsymbol{Y}, \mathscr{Y})$ be another approximative system map. We say that $f$ and $\bar{f}$ are simply approximatively equivalent, in notation $\boldsymbol{f}=: \bar{f}$, provided for each $b \in B$ there is an $a \geqslant f(b), \bar{f}(b)$ such that $\left(f_{b} p_{a, f(b)}, \bar{f}_{b} p_{a, \bar{f}(b)}\right) \leqslant \mathscr{V}_{b}$. We say that $f$ and $f$ are approximatively equivalent, in notation $\boldsymbol{f} \equiv: \hat{f}$, provided that there are finitely many approximative system maps $f_{1}, f_{2}, \ldots, f_{n}:(X, \mathscr{U}) \rightarrow(Y, \mathscr{V})$ such that $f=f_{1}, \bar{f}=f_{n}$ and $f_{i}=: f_{i+1}$ for $i=1,2, \ldots, n-1$. Trivially, $\equiv:$ is an equivalence relation and by $[f]$ we mean the equivalence class of $f$.

Let $(Z, \mathscr{W})=\left\{\left(Z_{c}, \mathscr{W}_{c}\right), r_{c^{\prime}, c}, C\right\}$ be an approximative inverse system and let $\boldsymbol{g}=\left\{g, g_{c}: c \in C\right\}:(\boldsymbol{Y}, \mathscr{V}) \rightarrow(\boldsymbol{Z}, \mathscr{W})$ be an approximative system map. Let $s$ : $C \rightarrow C$ be a function. We say that $s$ is a 1-refinement of $(Z, \mathscr{W})$ provided $s$ is an increasing function, $s(c) \geqslant c$ for $c \in C$ and $r_{s(c), c}^{-1}\left(\mathscr{W}_{c}\right) \geqslant \operatorname{st}\left(\mathscr{W}_{s(c)}\right)$ for $c \in C$. Here for $\mathscr{U} \in \operatorname{Cov}(X)$, we write st $(\mathscr{U})=\{\operatorname{st}(U, \mathscr{U}): U \in \mathscr{U}\} \in \operatorname{Cov}(X)$ and st $(U, \mathscr{U})=\bigcup\left\{U^{\prime} \in \mathscr{U}: U \cap U^{\prime} \neq \emptyset_{\}}\right.$. Any approximative inverse system has a 1-refinement function. We define the composition; $[h]=[g][f]$ of $[f]$ and [g] as follows: $\boldsymbol{h}=\left\{h, h_{c}: c \in C\right\}:(\boldsymbol{X}, \mathscr{U}) \rightarrow(\boldsymbol{Z}, \mathscr{W})$ is an approximative system map defined by $h=f g s$ and $h_{c}=r_{s(c), c} g_{s(c)} f_{g s(c)}$ for $c \in C$. We can prove that $[\boldsymbol{h}]$ is well defined, that is, $\boldsymbol{h}:(\boldsymbol{X}, \mathscr{U}) \rightarrow(\boldsymbol{Z}, \mathscr{W})$ is an approximative system map and $[h]$ does not depend on the 1-refinement function $s$ of $(Z, \mathscr{W})$ and on the representations of $[f]$ and $[g]$. We can show that this composition satisfies the associative law, and $[f]=[f]\left[\mathbf{1}_{\left(X, x_{1}\right)}\right]=\left[\mathbf{1}_{(Y, Y)}\right][f]$. Here $\mathbf{1}_{(X, \mathscr{U})}=\left\{1_{\boldsymbol{A}}, 1_{\boldsymbol{X}_{\boldsymbol{a}}}: a \in A\right\}:(X, \mathscr{U}) \rightarrow(\boldsymbol{X}, \mathscr{U})$. Hence, we can define the category of approximative pro-Top, in notation App(pro-Top), as follows: Objects are all approximative inverse systems and morphisms are all approximatively equivalent classes of all approximative system maps with the above composition.

We say that $p=\left\{p_{a}: a \in A\right\}: X \rightarrow(X, \mathbb{M})$ is an approximative resolution 
of a space $X$ provided $p_{a}: X \rightarrow X_{a}$ are maps, $p_{a}=p_{a^{\prime}, a} p_{a^{\prime}}$ for $a^{\prime} \geqslant a$ and the following two conditions are satisfied:

(AR1) For each $U_{\in} \operatorname{Cov}(X)$, there is an $a \in A$ such that $p_{a}^{-1}\left(\varkappa_{a}\right) \leqslant \|_{\text {. }}$

(AR2) For each $a \in A$ there is $a^{\prime} \geqslant a$ such that $p_{a^{\prime}, a}\left(X_{a^{\prime}}\right) \subset \operatorname{st}\left(p_{a}(X)\right.$, $\left.\|_{a}\right)$.

By ANR spaces we mean absolute neighborhood retracts for metric spaces (see Borsuk [2]). Polyhedra are simplicial complexes with the CWtopology. We say that $X$ is an approximative polyhedron, in notation AP,

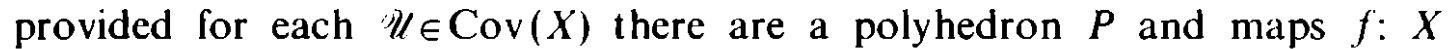
$\rightarrow P, g: P \rightarrow X$ such that $\left(g f, 1_{X}\right) \leqslant \mathscr{U}$ (see Mardešić [21]). ANRs and polyhedra are AP. We say that $p: X \rightarrow(X, U)$ is an approximative ANRresolution provided that it is an approximative resolution and all $X_{a}$ are ANRs. Similarly we use approximative AP-resolutions and approximative polyhedral resolutions. Mardešić [21] introduced the notions of resultion of spaces $p: X \rightarrow X$ and resolution of maps. Our notion is related to his notions. For an approximative inverse system $(X, \lambda)$ we can prove that $p: X$ $\rightarrow(X, U)$ is an approximative resolution if and only if $p: X \rightarrow X$ is a resolution. Mardešić [21] showed that any space admits an ANR-resolution. From this fact we can derive the conclusion that any space admits an approximative ANR-resolution and an approximative polyhedral resolution.

Let $q=\left\{q_{b}: b \in B_{\}}: Y \rightarrow(Y, \mathscr{Y})\right.$ be an approximative resolution and let $f: X \rightarrow Y$ be a map. We say that an approximative system map $f:(X, \mathscr{U})$ $\rightarrow(Y, \mathscr{V})$ is an approximative resolution of $f$ wih respect to $p$ and $q$ provided for each $b \in B,\left(f_{b} p_{f(b)}, g_{b} f\right) \leqslant y_{b}$. We can prove the following: If $\boldsymbol{p}$ and $\boldsymbol{q}$ are approximative AP-resolutions, then any map $f: X \rightarrow Y$ admits an approximative resolution $f:(X, \mathscr{I}) \rightarrow(Y, \mathscr{V})$ with respect to $p$ and $q$. In addition, if $\bar{f}:(X, \mathscr{U}) \rightarrow(Y, Y)$ is another approximative resolution of $f$ with respect to $p$ and $q$, then $f \equiv: \bar{f}$. Because of this we may denote by $[f]_{p, q}$ the approximative equivalence class containing the approximative resolution $f$ with respect to $p$ and $q$. We can prove that for maps $f: X \rightarrow Y$ and $g: Y$ $\rightarrow Z,[g]_{q . r}[f]_{p . q}=[g f]_{p . r}$ where $r: Z \rightarrow(Z, \mathscr{W})$ is any approximative AP resolution.

Let $p^{\prime}: X \rightarrow\left(X, \ell^{\prime}\right)^{\prime}$ and $q^{\prime}: Y \rightarrow\left(Y, y^{\prime}\right)^{\prime}$ be approximative AP resolutions and let $f^{\prime}:\left(X, \mathcal{U}^{\prime}\right)^{\prime} \rightarrow(\boldsymbol{Y}, \mathscr{Y})^{\prime}$ be an approximative system map. We say that $[f]$ and $\left[f^{\prime}\right]$ are equivalent, in notation $[f] \equiv\left[f^{\prime}\right]$, provided $\left[1_{Y}\right]_{q . q^{\prime}}[f]=\left[f^{\prime}\right]\left[1_{X}\right]_{p, p^{\prime}}$ in App(pro-Top). It is easy to show that $\equiv$ is an equivalence relation. By $\langle[f]\rangle$ we mean the equivalence class of $[f]$ by $\equiv$. Let $\boldsymbol{r}^{\prime}: Z \rightarrow\left(\boldsymbol{Z}, \mathscr{W}^{\prime}\right)^{\prime}$ be an approximative $\mathrm{AP}$ resolution and let $\boldsymbol{g}^{\prime}:(\boldsymbol{Y}, \mathscr{\boldsymbol { Y }})^{\prime}$ $\rightarrow(Z, \mathscr{W})^{\prime}$ be an approximative system map. We define the composition $\left\langle\left[g^{\prime}\right]\right\rangle\langle[f]\rangle$ by $\left\langle\left[g^{\prime}\right]\left[1_{Y}\right]_{q, q}[f]\right\rangle$. We can prove that this composition is well defined, satisfies the associative law and $\left.\langle[f]\rangle\left\langle 1_{X}\right]_{p . p}\right\rangle=\langle[f]\rangle$ $=\left\langle\left[1_{Y}\right]_{q . q}\right\rangle\langle[f]\rangle$. Hence, we can define the approximative shape category, in notation AppSh, as follows: Objects are all spaces, the set of morphisms 
from $X$ to $Y$ is the set $:\langle[f]\rangle: f:(X, \mathscr{Y}) \rightarrow(Y, \mathscr{Y})$ is an approximative system map and $p: X \rightarrow(X, 1 /), q: Y \rightarrow(X, Y)$ are approximative AP-resolutions?, with the above composition.

We define the approximative shape functor AS: Top $\rightarrow$ AppSh as follows: $\operatorname{AS}(X)=X$ for any space $X$ and $\operatorname{AS}(f)=\left\langle[f]_{p . q}\right\rangle$ for any map $f: X$ $\rightarrow Y$. Here $p$ and $q$ are approximative AP resolutions of $X$ and $Y$, respectively. Let $S h$ be the shape category and let $S:$ Top $\rightarrow$ Sh be the shape functor (see Mardešić and Segal [22]). We can define a functor ASS: AppSh $\rightarrow$ Sh such that $S=$ ASS AS. This means that many properties in AppSh imply shape properties.

Let Para and AppSh(Para) be full subcategories of Top and AppSh consisting of all paracompact Hausdorff spaces, respectively. We can prove that

(2.1) the functor AS induces an isomorphism of categories AS: Para $\rightarrow$ AppSh(Para).

(2.1) means that any map between paracompact spaces can be represented by an approximative resolution. This shows that question (1.3) has a positive answer, when we replace system maps by approximative system maps. Consequently, we can investigate bad spaces and bad maps by using the good categories Poly and ANR. Here Poly and ANR are the full subcategories of Top consisting of all polyhedra and all ANRs, respectively.

\section{§ 4. Applications}

Approximative shape has many applications in topology. For example, we studied generalized ANRs, fixed point theorems, shape fibrations, $U^{n}$-maps, hereditary shape equivalences, the Vietoris theorem in shape theory, Brown's near homeomorphism theorem (see [6]), Cech homology, Steenrod homology (see [29]), strong homology (see [19], [20]) and so on. In this section we explain our treatment of some of these topics.

There are many generalizations of ANRs. Noguchi [27] and Clapp [11] introduced approximative absolute neighborhood retracts $\mathrm{AANR}_{N}$ and $\mathrm{AANR}_{C}$, respectively. Granas [18] and Clapp [11] proved the LefschetzHopf fixed point theorem for compact metric $\mathrm{AANR}_{N}$ and $\mathrm{AANR}_{C}$, respectively. Borsuk $[3,4,5]$ introduced nearly extendable maps (NE-maps) and nearly exendable sets (Ne-sets) for compact metric space and proved the Lefschetz-Hopf fixed point theorem for NE-maps between compacta. Gauthier [15] extend Borsuk's definitions and results to compact Hausdorff spaces. Bogatyi [1] studied many properties of spaces including generalized ANRs. Mardešić [21] introduced approximate polyhedra (AP) and showed that $\mathrm{AP}$ and $\mathrm{AANR}_{C}$ coincide for compacta.

Our definition of approximative shape category is formally similar to the 
one of shape category. Therefore, we can introduce many notions in AppSh, which are analogous to some notions in shape. As an example, we now give the definition of approximative movability.

Let $(X, \mathscr{U})$ be an approximative inverse system. We say that it is approximatively movable provided for each $a \in A$ there exists an $a_{0} \geqslant a$ such that for each $a^{\prime} \geqslant a$ there is a map $r: X_{a_{0}} \rightarrow X_{a^{\prime}}$ satisfying $\left(p_{a^{\prime}, a} r, p_{a_{0}, a}\right) \leqslant \mathscr{U}_{a}$. We can prove that whenever $(Y, \mathscr{Y})$ is dominated by $(X, \mathscr{U})$ in App(pro-Top) and $(\boldsymbol{X}, \mathscr{U})$ is approximatively movable, then $(\boldsymbol{Y}, \mathscr{W})$ is also approximatively movable. We say that a space $X$ is approximatively movable provided it admits an approximative AP-resolution $p: X \rightarrow\left(X, \mathscr{U}^{\prime}\right)$ such that $(X, \mathcal{U})$ is approximatively movable. We can prove the following assertion: Let $p: X$ $\rightarrow(X, \mathscr{U})$ and $p^{\prime}: X \rightarrow(X, \mathscr{U})^{\prime}$ be approximative AP resolutions. Then $(X, \mathscr{U})$ is approximatively movable if and only if so is $(X, \mathscr{U})^{\prime}$. Hence, this notion does not depend on approximative AP-resolutions, and it is an invariant property in AppSh.

In a similar way we can introduce the notions of uniformly approximative movability, strongly approximative movability, ect. Using these new notions we can unify the various generalized ANRs in AppSh. Here we state some of the results:

(3.1) A compact metric space $X$ in an ANR if and only if it is strongly approximatively movable.

(3.2) A compact Hausdorff space $X$ is approximatively movable if and only if it is an NE-set.

(3.3) A compact Hausdorff space $X$ is uniformly approximatively movable if and only if it is an $\mathrm{AANR}_{C}$.

Next, we consider fixed point theorems. We say that $f:(X, \mathscr{U}) \rightarrow(X, \mathscr{Y})$ is nearly extendable provided for each $b \in B$ there is $a \geqslant f(b)$ such that for any $b^{\prime} \geqslant b$ there is a map $h: X_{a} \rightarrow Y_{b^{\prime}}$ satisfying $\left(f_{b} p_{a . f(b)}, q_{b^{\prime}, b} h\right) \leqslant \operatorname{st}\left(\mathscr{V}_{b}\right)$. We can prove the following assertion: Let $f: X \rightarrow Y$ be a map, let $p: X$ $\rightarrow(X, \mathscr{U}), p^{\prime}: X \rightarrow(X, \mathscr{U})^{\prime}, \boldsymbol{g}: Y \rightarrow(\boldsymbol{Y}, \mathscr{V})$ and $q^{\prime}: Y \rightarrow(\boldsymbol{Y}, \mathscr{V})^{\prime}$ be approximative AP resolutions and let $f:(X, \mathscr{U}) \rightarrow(Y, \mathscr{V})$ and $f^{\prime}:(X, \mathscr{U})^{\prime} \rightarrow(Y, \mathscr{V})$ be approximative resolutions of $f$ with respect to $p$ and $q$, and with respect to $\boldsymbol{p}^{\prime}$ and $q^{\prime}$, respectively. Then $f$ is nearly extendable if and only if so is $f^{\prime}$. From this fact, we say that $f$ is nearly extendable provided it admits an approximative resolution of $f$ with respect to some approximative AP-resolutions which is nearly extendable. We can prove that our definition of near extendability coincides with Borsuk's original definition.

Maxwell [24] proved a fixed point theorem for finite polyhedra. Masih [23] extended it to compact metric ANRs and Vora [30] to compact metric $\mathrm{AANR}_{N}$. Using only Maxwell's fixed point theorem for finite polyhedra, we can prove that 
(3.1) The Maxwell fixed point theorem holds for NE-maps on compact Hausdorff spaces.

As corollaries we obtain:

(3.2) The Lefschetz-Hopf fixed point theorem holds for NE-maps on compact Hausdorff spaces.

(3.3) The hyperspaces $2^{X}$ and $C(X)$ have the fixed point property for any approximatively movable compact Hausdorff space $X$.

(3.3) gives a partial answer to a question raised by Rogers [28]. (3.2) was proved by Borsuk [5] and Gauthier [15].

Morita [25] introduced the Cech homology based on all normal open coverings, and showed that this homology is a shape invariant. We showed that

(3.4) The Cech homology groups hased on normal open coverings satisfy all the axioms of Eilenberg-Steenrod except the exactness axiom, the wedge axiom and the relatice homeomorphism axiom.

Of course, the Cech cohomology based on all normal open coverings satisfies a theorem dual to (3.4). In general, Dowker [12] showed that Cech cohomology based on all open coverings (without assuming normality of the coverings) does not satisfy the relative homeomorphism axiom.

Finally, we summarize our point of view. The principle of shape theory is to investigate bad spaces and bad maps by means of the good categories HANR and HPoly. Here HANR and HPoly are the homotopy categories of ANR and Poly. On the other hand, our principle of approximative shape theory is to investigate bad spaces and bad maps by means of good calegories ANR and Poly. Cerin [7-10] takes a middle position. His principle is to investigate bad spaces and bad maps by means of small homotopy in ANR and Poly. Using small homotopies he introduces his notion of $C_{p}-e-$ movability and some others notions. Although our method does not use any homotopies, some of Cerin's notions coincide with some of ours.

\section{References}

[1] S. A. Bogatyi, Approximative and fundamental retracts, Math. Sbornik 93 (1974), 90-102.

[2] K. Borsuk, Theory of retracts, Warszawa 1967.

[3] -, On a class of compacta, Houston J. Math. 1 (1975), 1-13.

[4] -, On nearly exiendable maps, Bull. Acad. Polonaise 23 (1975), 753-760.

[5] -, On the Lefschetz-Hopf fixed point theorem for nearly extendable maps, ibid. 23 (1975), 1273-1279.

[6] M. Brown, Some applications of an approximation theorem for interse limits, Proc. Amer. Math. Soc. 11 (1969), 478-483.

[7] Z. Cerin, $C_{0}$-E-movahle and C-E-calm compacta and their images, Compositio Math. 45 (1981), 115-141. 
[8] -, C-E-morable and (C,D)-E-tame compacta, Houston J. Math. 9 (1983). 9.27.

[9] -, Strongly e-movable contergence and spaces of ANR's Topology Appl. 17 (1984), 27-46.

[10] -, Characterizing global properties in inverse limits. Pacific J. Math 12 (1984), 49. 68.

[11] M. H. Clapp, On a generalization of absolute neighborhood retracts, Fund. Math. 70 (1971), 117 130.

[12] C. H. Dowker, The excision theorem. Dokl. Akad. Nauk SSSR 125 (1959), 1190- 1192.

[13] S. Eilenberg and N. E. Steenrod, Foundations of algebraic topology, Princeteon Univ. Press, 1952.

[14] H. Freudenthal, Entwicklungen von Räumen und ihren Gruppen, Composition Math. 4 (1937), 145-234.

[15] G. Gauthier, Le théorème des points fixed de Lefichetz pur les NE-applications des espaces compacts non métrisables, Bull. Acad. Polonaise 28 (1980). 635-642.

[16] -, La theorie des retracts approximatifs et le théorème des points fixes de Lefschetz, Dissertation Math. 217 (1983), 1.82.

[17] A. Gmurczyk. On approximatice retacts, Bull. Acad. Polonaise 16 (1968) 9-14.

[18] A. Granas, Fixed point theorem for approximatice ANR-s, ibid. 16 (1968), 15-19.

[19] J. T. Lisitsa and S. Mardešić, Steenrod-Sitnikor homology for arhitrary spaces. Bull. Amer. Math. Soc. 9 (1983), 207 210.

[20] - -, Strong homology of inverse systems, I, II and III. Topology Appl. 19 (1985), 29 43; 45-64; and 20 (1985), 29-37.

[21] S. Mardešić, Approximate polyhedra, resolutions of maps and shape fibrations, Fund. Math. 114 (1981), 5387.

[22] S. Mardešić, and J. Segal, Shape theory, the inverse system approach, Nort-Holland Pub. Company, Amsterdam 1982.

[23] S. Masih, Fixed points of symmetric product mappings of polyhedra and metric absolute neighborhood retracts, Fund. Math. 80 (1973), 149-156.

[24] C. N. Maxwell, Fixed points of symmetric products mappings, Proc. Amer. Math. Soc. 8 (1957), 808-815.

[25] K. Morita, Cech cohomology and covering dimension for topological spaces, Fund. Math. 87 (1975). 3152.

[26] J. Mioduszewski, Mappings of inverse limits, Colloq. Math. 10 (1963), 39.44.

[27] H. Noguchi, A generalization of absolute neighborhood retracts, Kodai Math. Seminar 1 (1953), 20-22.

[28] J. T. Rogers, Jr., The cone = hyperspace property, Canad. J. Math. 24 (1972), 279- 285.

[29] N. E. Steenrod, Regular cycles of compact metric spaces, Ann. of Math. 41 (1940), 833851.

[30] C. Vora, Fixed points of certain symmetric product mappings of metric manifold, Fund. Math. 85 (1974), 1924.

[31] T. Wat a nabe, Approximative shape theory, Ch.l-Ch.V, mimeographed notes, University of Yamaguchi 1981-1984.

[32] -, Cech homology, Steenrod homology and strong homology, to appear in Glasnik Mat.

Presented to the Topology Semester

April 3 - June 29, 1984 